\title{
Effect of the shape of imprinted alignment layer on the molecular orientation of liquid crystal
}

\author{
Hyundae Hah, Shi-Joon Sung, Manhee Han ${ }^{\text {a, }}$, Seungsup Lee ${ }^{\text {a,1 }}$, Jung-Ki Park ${ }^{\text {b,* }}$ \\ ${ }^{a}$ Department of Mechanical Engineering, Korea Advanced Institute of Science and Technology, 373-1, Guseong-Dong, Yuseong-Gu, Daejon, 305-701, Korea \\ ${ }^{\mathrm{b}}$ Department of Chemical and Biomolecular Engineering, Korea Advanced Institute of Science and Technology, 373-1, \\ Guseong-Dong, Yuseong-Gu, Daejon, 305-701, Korea
}

Received 23 March 2006; received in revised form 22 August 2006; accepted 27 August 2006

Available online 4 October 2006

\begin{abstract}
The molecular alignment of liquid crystals was studied with three different imprinted patterns. The three different shapes of molds (square-, Vand U-shaped) replicated the microstructures with electroplating process was fabricated. Surfaces with three different microgrooved patterns were used as the alignment layer of the liquid crystal cell. The effect of the shape of imprinted alignment layer on the molecular orientation of LC was investigated. Among these patterns, U-shaped groove pattern resulted in a high performance of liquid crystal orientation, pretilt angle and anchoring energy, which are quite comparable to the conventionally rubbed layer. It clearly shows that the molecular orientation of liquid crystals is influenced by the shape of microgrooved patterns.
\end{abstract}

(C) 2006 Elsevier B.V. All rights reserved.

Keywords: Imprinted LC alignment layers; Electroplating process; Pretilt angel; Anchoring energy

\section{Introduction}

The alignment phenomenon of liquid crystal molecules on a solid surface and the technique of the alignment control have been an attractive subject for liquid crystal display application. Usually, liquid crystal alignment is induced by the rubbing method $[1,2]$. The rubbed polymer surface has optical anisotropies caused by the orientation of polymer molecules and microgrooves, which is responsible for the LC alignment [3]. However, the rubbing process has some problems such as generation of static charge, dust and scratches on the surface of alignment layer. To overcome the demerit of rubbing method, many studies have been done on the use of the replicated patterns for the liquid crystal alignment [4-9]. Scharf et al. investigated the LC alignment properties with UV embossing techniques on the replicated surfaces [8]. Also, the liquid crystal orientation

\footnotetext{
* Corresponding author. Tel.: +82 42869 3925; fax: +82 428693910. E-mail address: jungpark@kaist.ac.kr (J.-K. Park).

1 Tel.: +82 42869 3046; fax: +82 428693920 .
}

with the line pitch for the given edge shape was studied with liquid crystal alignment by photolithographic microgrooved patterns [9]. Unfortunately, however, there have been no results that are completely successful for the preparation of the replicated patterns for the practical application of liquid crystal alignment. It could be postulated that the shape of imprinted alignment layer influences on the molecular orientation of LC. The molecular orientation of liquid crystal is controlled by the shape of surface, the elasticity of liquid crystal, and the interaction energy. However, the influence of shape of microgrooved patterns on the properties of liquid crystal was not yet addressed.

In the present work, we tried to prepare various shapes (square-, V- and U-shaped) of imprinted grooves which might produce different liquid crystal alignment properties. We demonstrated the fabrication of three shapes of imprinted LC alignment layers with different scale by electroplating process to enhance the LC orientational behavior. We investigated the effect of the shape of imprinted alignment layer on the molecular orientation of LC. We suggest our approaches are very promising for the practical application of the imprinted patterns to the liquid crystal device. 


\section{Experimental}

Polyimide derived from 4,4'-(hexafluoro-isopropylidene) diphthalic anhydride and 4,4'-oxydianiline (6FDA-ODA) was synthesized with general methods by using monomers in Fig. 1 [10]. Polyamic acid was thermally imidized during the thermal curing of the spin-coated cast.

The square-, U- and V-shaped molds were fabricated by replicating the square-, U- and V-shaped photoresist microstructures with electroplating process, respectively. The squareshaped photoresist microstructure is fabricated using a photolithography process including a spin-coating step, an exposing step and a developing step [11]. The U-shaped one is fabricated using the photolithography process and a reflow method [12]. The V-shaped one is fabricated using an inclined lithography process in which UV light is inclined in the exposing step [13]. On the surface of the microstructured photoresist, titanium is deposited as a seed layer for a nickel electroplating process. After the electroplating process, the final metal molds are completely formed by removing other materials except for nickel. The depth and width of groove of each pattern are $3 \mu \mathrm{m}$.

Alignment layers were prepared by spin coating on the slide glass with a polyamic acid solution in NMP and thermally imidized.

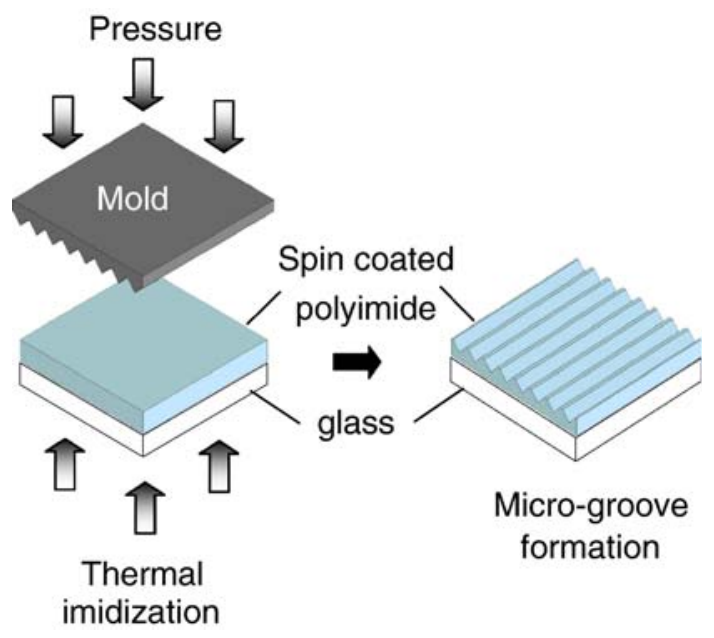

Fig. 2. Schematic illustration of making microgrooves on polyimide surfaces by imprinting method with molds.

A homogeneously aligned LC cell was constructed by sandwiching nematic LC (E7) between a couple of glass substrates. The thickness of the liquid crystal layer was adjusted by tiny spherical spacers (polystyrene) of $8 \mu \mathrm{m}$ diameter. The texture of liquid crystal in the cell was investigated with Nikon OPTOPHOTO2-POL<smiles>Cc1ccc(Oc2ccc(N)cc2)cc1</smiles><smiles>Cc1ccc(Oc2ccc(NC(=O)c3cc(C(F)(F)C(F)(F)F)ccc3C(=O)O)cc2)cc1</smiles>

Poly (amic acid)

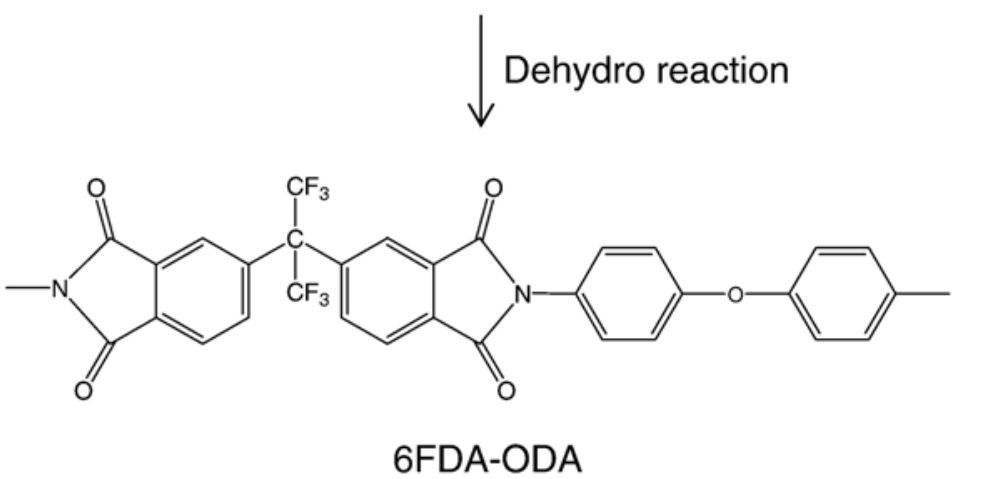

Fig. 1. Synthesis of 6FDA-ODA. 


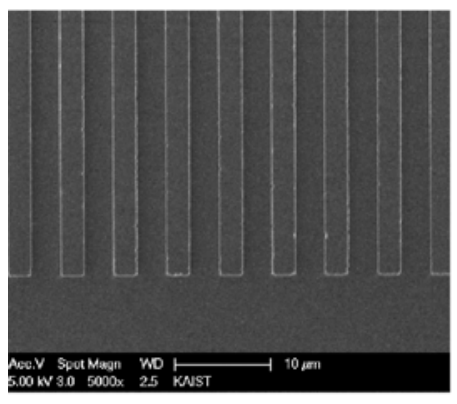

(a) Square-grooved mold surface

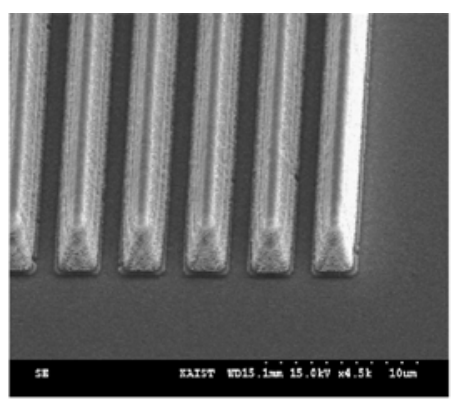

(b) V-grooved mold surface

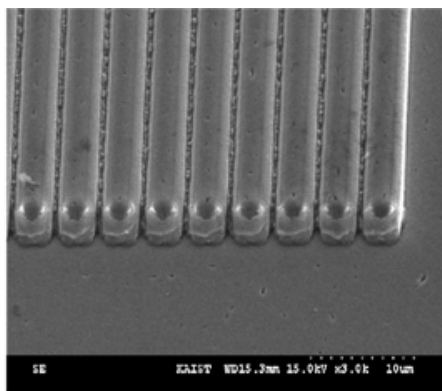

(c) U-grooved

mold surface

Fig. 3. Images of scanning electron microscopy for the three shapes of microgrooved molds surfaces: (a) square-grooved, (b) V-grooved and (c) U-grooved molds surfaces.

polarized optical microscopy under cross Nicoles. LC cells were prepared to measure the pretilt angles, azimuthal anchoring energies and order parameter of LC. We used crystal rotation method to measure pretilt angle [14]. The azimuthal anchoring energies were estimated by the measurement of the width of Neel wall [15]. The order parameter of the LC cell was measured using one polarizer and UV-vis spectrometer. Spectral measurements of UV-vis were performed by using a Shimadzu UV-1601.

\section{Results and discussion}

We fabricated three different shaped-molds and made microgrooves on polyimide surfaces by imprinting method with molds, which is schematically described in Fig. 2. This approach is convenient and rapid to pattern polyimide surfaces. Fig. 3 shows images of scanning electron microscopy for the three different shapes of microgrooved molds surfaces. Fig. 4 represents the polyimide surfaces after removing the three different shapes of microgrooved molds.

To elucidate the effect of shape of the imprinted alignment layer on the molecular orientation of LC, we examined order parameter, pretilt angles and azimuthal anchoring energies of the LC molecules on the surface of each imprinted alignment layer. The direction of LC orientation can usually be represented by the order parameter. The order parameter of LC from the square-shaped polyimide LC alignment layer was 0.42 . Pretilt angle of LC was measured by means of crystal rotation method. From the transmittance as a function of the incident angle of $\mathrm{He}-\mathrm{Ne}$ laser beam as a probing light passed through a cell set between two crossed polarizers, pretilt angle of LC on the square-shaped polyimide LC alignment layers was $3.5^{\circ}$. For the azimuthal anchoring energy, the width of the Neel wall, $\omega$, is defined as the distance between two black brushes where the director is rotated from $45^{\circ}$ to $135^{\circ}$ with respect to the director in a uniformly aligned region. The azimuthal anchoring energy is then calculated using the following formula

$E_{\Phi}=2 d K_{1} / \omega_{2}$

where $K_{1}$ and $d$ represent the elastic constant for splay deformation and the thickness of LC layer, respectively. The azimuthal anchoring energy of LC on the square-shaped polyimide LC alignment layers was calculated to be $7.104 \times 10^{-6} \mathrm{~J} / \mathrm{m}^{2}$. The orientation of $\mathrm{LC}$ molecules depends on the width of groove and ridge between grooves. In the rubbing process, the microscopic grooves with small ridge have interaction with liquid crystal, which means that there are orientation domains in whole area. In the square-shaped alignment layer, the ridge $(3 \mu \mathrm{m})$ between grooves having not aligned liquid crystal is wider than the width of groove having

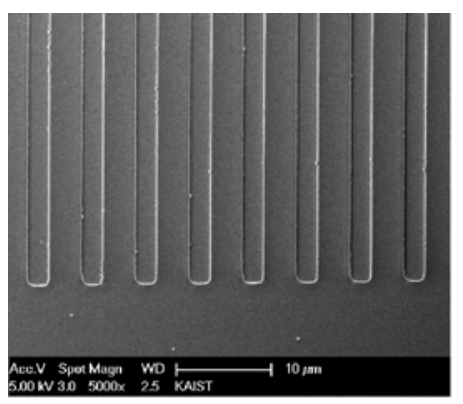

(a) Square-grooved polyimide LC alignment layer

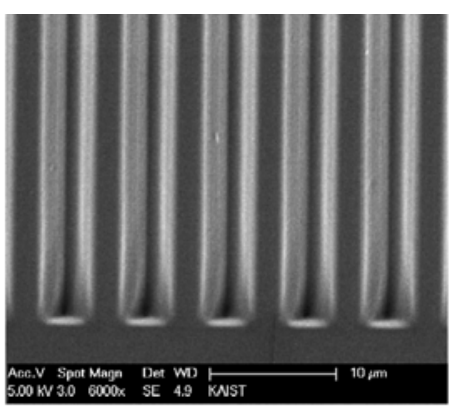

(b) V-grooved polyimide LC alignment layer

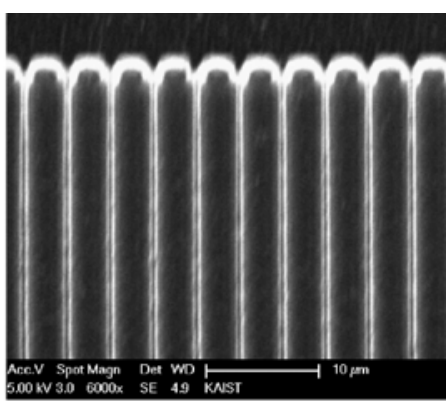

(c) U-grooved polyimide LC alignment layer

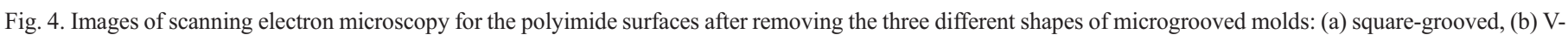
grooved and (c) U-grooved polyimide LC alignment layers. 
Table 1

The order parameter, pretilt angles and azimuthal anchoring energies of LC from three different grooved and rubbed alignment layers

\begin{tabular}{llll}
\hline & $\begin{array}{l}\text { Order } \\
\text { parameter }\end{array}$ & $\begin{array}{l}\text { Pretilt } \\
\text { angle }\end{array}$ & $\begin{array}{l}\text { Azimuthal } \\
\text { anchoring energies }\end{array}$ \\
\hline $\begin{array}{c}\text { Square-grooved polyimide } \\
\text { LC alignment layers }\end{array}$ & 0.42 & $3.5^{\circ}$ & $7.104 \times 10^{-6} \mathrm{~J} / \mathrm{m}^{2}$ \\
$\begin{array}{c}\text { V-grooved polyimide } \\
\text { LC alignment layers }\end{array}$ & 0.46 & $5.5^{\circ}$ & $2.841 \times 10^{-5} \mathrm{~J} / \mathrm{m}^{2}$ \\
$\begin{array}{c}\text { U-grooved polyimide } \\
\text { LC alignment layers }\end{array}$ & 0.53 & $8.3^{\circ}$ & $1.138 \times 10^{-4} \mathrm{~J} / \mathrm{m}^{2}$ \\
$\begin{array}{c}\text { Rubbed LC alignment } \\
\text { layer }\end{array}$ & 0.63 & $8.5^{\circ}$ & $5.4 \times 10^{-3} \mathrm{~J} / \mathrm{m}^{2}$ \\
\hline
\end{tabular}

aligned liquid crystal. This represents that the orientation domain is small comparing with rubbed layer. Also, the rectangular shape of square-groove interrupts $\mathrm{LC}$ to have continuous flow on the inside of square-groove. It seems to be hard for liquid crystal to have high pretilt angle and azimuthal anchoring energy on the square-shaped alignment layer, comparing with rubbed layer.

In the case of V-shaped polyimide LC alignment layers, the order parameter of $\mathrm{LC}$ was 0.46 . The order parameter of $\mathrm{LC}$ was a little increased compared with that from the square-shaped polyimide alignment layers. The pretilt angle and the azimuthal anchoring energy of LC on the V-shaped polyimide LC alignment layers were increased to $5.5^{\circ}$ and $2.841 \times 10^{-5} \mathrm{~J} / \mathrm{m}^{2}$, respectively. In the V-shaped alignment layer, liquid crystal is aligned according to the width of grooves and the ridge $(2.5 \mu \mathrm{m})$ between grooves having not aligned liquid crystal is narrower than squaregroove. The orientation domain in the $\mathrm{V}$-shaped alignment layer is larger than that in the square-shaped alignment layer, which resulted in the enhancement of alignment properties of liquid crystals in the V-shaped alignment layer. However, the sharpness of the edge of groove could disturb to have continuous flow of liquid crystal on the inside of $\mathrm{V}$-groove. It was hard for the Vshaped alignment layer to have high pretilt angle and azimuthal anchoring energy, comparing with rubbed layer.

For the U-shaped polyimide LC alignment layers, the order parameter of $\mathrm{LC}$ was 0.53 . The order parameter was significantly higher compared with that from the square-shaped polyimide LC alignment layers. The pretilt angle in the U-shaped polyimide LC alignment layers was increased to $8.3^{\circ}$. The pretilt angle on the $\mathrm{U}$-shaped polyimide LC alignment layers was higher than that of nanostructured surfaces because U-shaped polyimide LC alignment layers had high azimuthal anchoring energy. We found that the U-shaped polyimide LC alignment layers showed also the highest azimuthal anchoring energy $\left(1.138 \times 10^{-4} \mathrm{~J} / \mathrm{m}^{2}\right)$ of LC among three different shaped polyimide LC alignment layers. The anchoring energy for the U-shaped polyimide LC alignment layers is about one order of magnitude lower than for rubbed alignment layer. This is close to the value available from the rubbed layer. The orientation of the liquid crystal on the U-shaped alignment layer follows the contours on the inside of grooves. The ridge $(1 \mu \mathrm{m})$ between grooves having not aligned liquid crystal is narrower than square- and V-groove. This represents that the orientation domain in the U-shaped alignment layer is much larger than that in the square- and V-shaped alignment layer, which resulted in the enhancement of alignment properties of liquid crystals in the U-shaped alignment layer. Accordingly as the ridge between grooves decreased, the alignment properties of liquid crystal were enhanced. It is found that the orientation of LC molecules is determined by the width of groove with narrow ridge between grooves. Also, the smoothness of the edge of groove helps to have continuous flow of liquid crystal on the inside of U-groove, which resulted to have high pretilt angle and azimuthal anchoring energy. Those properties in the U-shaped polyimide LC alignment layers were close to those in rubbed layer. The order parameter, pretilt angles and azimuthal anchoring energies of LC from the three different shaped-and rubbed alignment layers are summarized in Table 1.

From the above results, it is evident that the shape of grooved alignment layer has a significant influence on the molecular orientation of LC. It seems that smoothness of the groove for the continuous flow of LC and the ridge between the grooves influence the LC alignment behavior very significantly; however, the details of these phenomena need to be studied further. This investigation is believed a good approach to understand the alignment behavior of liquid crystal molecules.

\section{Conclusion}

We investigated the effect of the three different shape of imprinted alignment layer on the molecular orientation of LC. The different LC alignment behaviors were shown with three different patterns. Among these patterns, U-shaped groove pattern resulted in a high performance of liquid crystal orientation, pretilt angle and anchoring energy, which are quite comparable to the conventionally rubbed layer. It seems that smoothness of the groove for the continuous flow of LC and the ridge between the grooves influences the $\mathrm{LC}$ alignment behavior very significantly. It is found that the shape of grooved alignment layer has a significant influence on the molecular orientation of LC. Our approach is expected to be promising for practical application of imprinting method to liquid crystal alignment.

\section{References}

[1] J.V. Haaren, Nature 411 (2001) 29.

[2] M. Suzuki, T. Maruno, F. Yamamoto, K. Nagai, J. Vac. Sci. Technol., A 8 (1990) 631.

[3] D.W. Berreman, Phys. Rev. Lett. 28 (1972) 1683.

[4] D.W. Berreman, Mol. Cryst. Liq. Cryst. 23 (1973) 215.

[5] J. Cheng, G.D. Boyd, Appl. Phys. Lett. 35 (1979) 444.

[6] M. Kim, J. Kim, Liq. Cryst. 27 (2000) 1633.

[7] M. Nakamura, M. Ura, J. Appl. Phys. 52 (1981) 210.

[8] T. Scharf, A. Shlayen, C. Gernes, N. Basturk, J. Grupp, Mol. Cryst. Liq. Cryst. 411 (2004) 1745 .

[9] Y. Kawata, K. Takatoh, M. Hasegawa, M. Sakamoto, Liq. Cryst. 16 (1994) 1027.

[10] S. Sung, J. Lee, K. Cho, W. Kim, H. Hah, H. Shim, J. Park, Liq. Cryst. 31 (2004) 1601.

[11] M.J. Madou, CRC press (2001).

[12] F.T. O’Neill, J.T. Sheridan, Optik 113 (2002) 391.

[13] H. Sato, T. Kakinuma, J.S. Go, S. Shoji, Proc. IEEE Micro Electro Mech. Syst., 2003, p. 223.

[14] T.J. Scheffer, J. Nehinng, J. Appl. Phys. 48 (1977) 1783

[15] Y. Imura, Y. Kobayshi, T. Hashimoto, T. Sugiyama, K. Katoh, IEICE Trans. ELECTRON E79-C, 1996, p. 1040. 